\title{
Evaluation of Sleep Quality and Psychological Profiles in Patients with Chronic Painful Temporomadibular Disorders
}

\author{
Hye-Mi Jeon ${ }^{1}$, Kyung-Hun Han ${ }^{2}$, Hye-Min Ju', Yong-Woo Ahn ${ }^{3}$, Soo-Min Ok ${ }^{3}$, Sung-Hee Jeong ${ }^{3}$ \\ 1'Dental Clinic Center, Pusan National University Hospital, Busan, Korea \\ ${ }^{2}$ Division of Sport Science, Pusan National University, Busan, Korea \\ ${ }^{3}$ Department of Oral Medicine, Pusan National University School of Dentistry, Yangsan, Korea
}

Received June 3, 2020

Revised June 14, 2020

Accepted June 14, 2020

\section{Correspondence to:}

Sung-Hee Jeong

Department of Oral Medicine, Pusan

National University School of Dentistry, 49

Busandaehak-ro, Mulgeum-eup, Yangsan

50612, Korea

Tel: $+82-55-360-5242$

Fax: +82-55-360-5238

E-mail:drcookie@pusan.ac.kr

https://orcid.org/0000-0002-6296-4775

This study was supported by Clinical Research Grant from Pusan National University Hospital in 2020.
Purpose: Temporomadibular disorders (TMDs) can result in chronic pain, which is often associated with psychological and sleep disturbance. Increased levels of psychological and sleep impairments are often related with poor treatment outcomes. The purpose of the present study was to evaluate clinical features, psychological profiles and the prevalence of clinical insomnia in TMD patients with chronic pain and to suggest an effective treatment approach.

Methods: A total of 200 TMD patients who had visited the Pusan National University Hospital dental clinic for treatment of their pain were recruited from June 2018 through December 2019. TMD patients were classified into an acute $(n=100)$ and chronic $(n=100)$ group and compared the clinical symptoms. The primary diagnosis of TMD were categorized as TMD with joint pain group (TMD_J), TMD with muscle pain group (TMD_M) and TMD with joint-muscle mixed pain group (TMD_JM). Self- report measures of sleep quality and psychological profiles were evaluated via the Insomnia Severity Index (ISI) and the Korean Stress Response Inventory (SRI). Independent t-tests, Mann-Whitney U-tests, and chisquared test were used for the statistical analysis.

Results: Chronic TMD patients showed higher pain intensity, as well as higher prevalence of related symptoms (headache, musculoskeletal pain) and myogenous pain. They also had significantly higher scores in all SRI parameters and a higher percentage of clinical insomnia than acute TMD patients.

Conclusions: Based on the above results, psychological profiles and sleep quality assessments are necessary to provide essential data that will allow for improved treatment of chronic TMD patients.

Key Words: Chronic pain; Psychological stress; Sleep; Temporomadibular disorders

\section{INTRODUCTION}

Temporomandibular disorders (TMDs) are embrace distinguished clinical problems involving the masticatory muscles, the temporomandibular joint (TMJ), or both [1]. Although the exact causes of the most TMDs remain either largely unknown or speculative, most are assumed to be due to many factors, including trauma, anatomical, pathophysiological, social, and psychological factors. The chief clinical symptom of most TMDs is pain, and TMD patients often complain of increased pain sensitivity and psychological dysfunction due to its chronic nature. This emphasizes the importance of gaining information about the duration of the patient's pain complaints. Patient with severe psychological stress-related problems as well as pain should have both managed simultaneously, because failure

Copyright (c) 2020 Korean Academy of Orofacial Pain and Oral Medicine. All rights reserved.

(c) This is an open-access article distributed under the terms of the Creative Commons Attribution Non-Commercial License (http://creativecommons.org/licenses/by-nc/4.0/), which permits unrestricted non-commercial use, distribution, and reproduction in any medium, provided the original work is properly cited. 
to resolve the severe psychological stress-related reactions can interfere with the appropriate treatment of the TMD pain $[2,3]$. There has been continued attention to the study of the relationship between psychological stress and TMD. In this regard, various attempts have been made to measure psychological stress in a quantifiable manner. In spite of being criticized for their subjectivity, self-rating reports have been a primary modality for measuring stress in clinic settings [4].

Insomnia, defined as "a persistent difficulty with sleep initiation, duration, consolidation, or quality that occurs despite adequate opportunity and circumstances for sleep", has long been associated with chronic pain and psychological distress [5]. Fibromyalgia, one of the notorious chronic pain, showed high rates of pain, poor sleep and depression. Sleep difficulties were reported by 77\% of patients with orofacial pain, including TMD patients. Thus, it is clinically important to identify chronic TMD patient with a high risk for insomnia and sleep evaluations are essentials for the proper assessment and treatment of chronic TMD [6]. However, dental clinics do not normally have the resources or expertise to provide detailed sleep assessments for TMD patients with insomnia. To evaluate insomnia, subjective assessments are considered the gold standard, and have been recommended for the evaluation of patients with TMD [7-9].

Several studies has evaluate the clinical, psychological profiles and sleep disturbance in the TMD patients have been reported $[1,2,6]$. Unfortunately, there are limited studies regarding the chronicity of TMD pain. Therefore, this study was designed (1) to assess psychological profiles and the prevalence of clinical insomnia based on self-report questionnaires and to compare the differences in clinical characteristics in TMD patients; (2) to propose an effective management approach for chronic TMD patients.

\section{MATERIALS AND METHODS}

\section{Subjects}

TMD patients who had visited the Pusan National University Hospital dental clinic for treatment of their pain were recruited from June 2018 through December 2019. Exclusion criteria were as follows (1) patients with history of facial and jaw fracture, and orthognathic surgery, (2) patients who had a history of psychiatric conditions, (3) patients who had neurologic impairment or diseases (e.g., stroke, tumor, trauma, or epilepsy), (4) patients who had other systemic muscular disorders (e.g., fibromyalgia, inflammatory joint disease), (5) patients who were pregnant. A total of two-hundred TMD patients were classified by acute and chronic group. Chronicity was established by persistence of pain beyond 3 months of symptoms. The research protocol was approved by the Ethics Committee of Pusan National University Hospital (PNUH; approval no. H-1806-007-067) and informed consent was obtained.

\section{Clinical Signs and Symptoms}

Each subjects examined their clinical signs and symptoms based on the Research Diagnostic Criteria for TMD axis I guidelines including pain intensity, comfortable mouth opening (CMO), pain duration and related symptoms (headache, comorbid musculoskeletal pain, sleep disturbance). Subjects were divided into the patients with joint pain group (TMD_J), patients with muscle pain group (TMD_M) and patients with joint-muscle mixed pain group (TMD_JM).

\section{Questionnaires}

Psychological profile and sleep quality were assessed with self-report instruments administered at the initial evaluation. The questionnaires were consisted of two parts;

\section{1) Insomnia Severity Index}

Insomnia Severity Index (ISI) is a self-rating scale designed to assess the subjective perception of the severity of insomnia. ISI has been translated into several languages and used to evaluate insomnia in pain research [10]. A Korean version of the ISI was validated in 2014 by Cho et al. [11]. The questionnaire contains seven items assessing: (1) the severity of sleep-onset, (2) sleep maintenance, (3) early morning awakening problems, (4) satisfaction with current sleep pattern, (5) interference with daily functioning, (6) noticeability of impairment attributed to the sleep problem, and (7) level of distress caused by the sleep problem. Patients rated each items on a 5-point scale (not at all, 0; Somewhat, 1; Moderately, 2; Very much, 3; Absolutely, 
4), and total scores range from 0 to 28 , with higher scores indicating more severe insomnia. According to the recommended score interpretation guideline, a total score of 0-7 indicates 'no clinically significant insomnia', 8-14 indicates 'sub-threshold insomnia', 15-21 indicates 'moderate clinical insomnia', and 22-28 indicates 'severe clinical insomnia' A cutoff score of 15 has been used as the threshold for clinically significant insomnia, and a score below 8 has been used to define remission after treatment.

\section{2) Stress Response Inventory}

Psychological profiles were assessed based on the Stress Response Inventory (SRI). SRI is validate assessment method that has been developed by Ko et al. [4] in Korean to measure of stress levels for research in stress-related field within a short time. It includes seven symptom dimensions: tension subscale (6 items), aggression subscale (4 items), somatization subscale (3 items), anger subscale (6 items), depression subscale (8 items), fatigue subscale ( 5 items), and frustration subscale (7 items). In total, the questionnaire contained 39 items, each of which was arranged in a Likerttype format: not at all, 0; Somewhat, 1; Moderately, 2; Very much, 3; Absolutely, 4. The global score ranges from 0 to 156 , with higher scores indicating more severe stress responses.

\section{Statistical Analysis}

A normality test for the parameters was performed using the Kolmogorov-Smirnov test. For comparison of the differences between the acute and chronic TMD groups, data were analyzed by the independent t-test or the MannWhitney U-test for continuous variables. Chi-square test was used for assessing differences in categorical variables between two groups. Statistical tests were done at the 5\% significance level. The data were analyzed using IBM SPSS Statistics for Windows, Version 22.0 (IBM Co., Armonk, NY, USA).

\section{RESULTS}

\section{Comparison of Demographic and Clinical Features}

The clinical and descriptive data were compared between acute and chronic group. As presented in Table 1, there were no significant differences between two groups for age and CMO. However chronic group showed significantly higher pain intensity and longer pain duration. The prevalence of acute TMD was about 2 times higher in women (62.0\%) than in men (38.0\%). In chronic group, women (83.0\%) showed about 5 times higher prevalence than men (17.0\%). The distribution of TMD patients according to the primary diagnostic group differed significantly between acute and chronic group $(\mathrm{p}<0.001)$. The percentage of the TMD_M and TMD_JM were significantly higher in chronic group than acute group. Table 2 shows the differences in the prevalence of related symptoms between two groups. Chronic group reported higher prevalence of headache and

Table 1. Demographics and clinical characteristics of the patients

\begin{tabular}{|c|c|c|c|}
\hline Variable & Acute $(n=100)$ & Chronic $(n=100)$ & $p$-value \\
\hline Age (y) & $41.3 \pm 17.5$ & $36.8 \pm 17.7$ & $0.067^{\mathrm{a}}$ \\
\hline Pain intensity & $3.5 \pm 1.2$ & $4.4 \pm 1.9$ & $<0.001^{\mathrm{a}}$ \\
\hline $\mathrm{CMO}(\mathrm{mm})$ & $35.5 \pm 8.5$ & $35.1 \pm 7.4$ & $0.698^{\mathrm{a}}$ \\
\hline Pain duration (mo) & $0.7 \pm 0.6$ & $28.4 \pm 49.3$ & $<0.001^{\mathrm{a}}$ \\
\hline $\operatorname{Sex}(\%)$ & & & $0.001^{b}$ \\
\hline Male & 38.0 & 17.0 & \\
\hline Female & 62.0 & 83.0 & \\
\hline Diagnosis (\%) & & & $<0.001^{\mathrm{b}}$ \\
\hline TMD_J & 72.0 & 43.0 & \\
\hline TMD_M & 9.0 & 19.0 & \\
\hline TMD_JM & 19.0 & 38.0 & \\
\hline
\end{tabular}

CMO, comfortable mouth opening; TMD, temporomadibular disorder; TMD_J, joint pain group; TMD_M, muscle pain group, TMD_JM, jointmuscle mixed pain group.

Values are presented as mean \pm standard deviation.

${ }^{a} \mathrm{p}$-values were determined from independent t-test. ${ }^{b} \mathrm{p}$-value was determined from Chi-square test. 
Table 2. Differences in the prevalence of related symptoms

\begin{tabular}{lccr}
\hline Variable & $\begin{array}{c}\text { Acute } \\
(n=100)\end{array}$ & $\begin{array}{r}\text { Chronic } \\
(n=100)\end{array}$ & p-value \\
\hline Headache & 41 & 63 & 0.002 \\
Comorbid musculoskeletal pain & 36 & 68 & $<0.001$ \\
Sleep disturbance & 17 & 27 & 0.088 \\
\hline
\end{tabular}

Values are presented as the number of subjects.

$\mathrm{p}$-values were determined from Chi-square test.

Table 3. Comparison of the ISI scores

\begin{tabular}{cccc}
\hline IS| & Acute $(n=100)$ & Chronic $(n=100)$ & $p$-value \\
\hline 0 & 63 & 51 & 0.049 \\
1 & 27 & 33 & \\
2 & 10 & 13 & \\
3 & 0 & 3 & \\
\hline
\end{tabular}

ISI, Insomnia Severity Index.

Values are presented as the number of subjects.

p-values were determined from Chi-square test.

comorbid musculoskeletal pain than acute group. There was no significant differences in the prevalence of sleep disturbance due to pain between two groups.

\section{Comparison of the Insomnia Severity Index Scores}

ISI scores of two groups were presented in Table 3. In acute group, 37\% patients reported mild to severe insomnia symptoms (ISI score $\geq 8$ ) and clinically significant insomnia (ISI score $\geq 15$ ) was observed in $10 \%$ of patients. Chronic group presented significantly higher prevalence (49\% and $16 \%$, respectively) than acute group ( $\mathrm{p}=0.049$ ).

\section{Psychological Profiles}

There was a significant difference in SRI score between chronic and acute TMD patients. As shown in Table 4, chronic group reported significantly high scores than acute group in all subscales (tension, aggression, somatization, anger, depression, fatigue, frustration) as well as total score.

\section{DISCUSSION}

TMD cases with chronic pain require a thorough psychological diagnostic assessment. Such an assessment is necessary to determine if the patient's chronic pain is related to depression, somatization, or other psychosocial conditions [12]. Smith [13] reviewed the relationship between
Table 4. Differences in SRI scores of patients

\begin{tabular}{lccr}
\hline SRI & Acute $(n=100)$ & Chronic $(n=100)$ & $p$-value \\
\hline T & $2.9 \pm 3.8$ & $4.4 \pm 4.0$ & $<0.001$ \\
Ag & $0.4 \pm 1.7$ & $0.9 \pm 2.2$ & 0.046 \\
S & $1.5 \pm 2.0$ & $2.6 \pm 2.2$ & $<0.001$ \\
An & $2.9 \pm 3.7$ & $4.3 \pm 4.3$ & 0.011 \\
D & $3.7 \pm 5.1$ & $5.6 \pm 5.7$ & 0.004 \\
F & $3.6 \pm 3.3$ & $5.7 \pm 4.1$ & $<0.001$ \\
Fr & $3.8 \pm 4.9$ & $6.6 \pm 6.1$ & $<0.001$ \\
Total & $18.6 \pm 22.0$ & $30.1 \pm 24.5$ & $<0.001$ \\
\hline
\end{tabular}

SRI, Stress Response Inventory; T, tension; Ag, aggression; $S$, somatization; An, anger; D, depression; F, fatigue; Fr, frustration.

Values are presented as mean \pm standard deviation was tested with the independent t-test.

p-values were determined from Mann-Whitney U-test.

depression and pain, and reported that chronic pain increases the risk of affective disturbances including depression, and vice versa. Fishbain et al. [14] demonstrated that depression is more common in chronic pain patients than in healthy controls due to the presence of chronic pain. In this study, chronic TMD patients showed significantly higher psychological scores in all parameters compared to acute TMD patients. Several studies have found that women are more likely to become depressed than men are $[4,15,16]$. Interestingly, women were significantly more prevalent in the chronic group than in the acute group in this study. We speculated that this might be due to the predominance of psychological problems in the chronic group.

The presence of chronic pain has the potential to induce long lasting neuroplastic changes of the peripheral and central nervous system. Hertig et al. [17] reported that patients with fibromyalgia demonstrate clinical evidence of a centrally mediated allodynia and general nociceptive facilitation, which yields higher pain intensity to painful pressure stimuli. Initial single-site chronic pain may cause central sensitization, thereby increasing the risk of experiencing pain in other body regions. TMD can itself become chronic as a result of central mediation and sometimes be a regional manifestation of a widespread centrally medicated chronic pain phenomenon such as fibromyalgia [12]. Park et al. [18] suggested that chronic TMD patients have peripheral and central sensitization. Our study showed that not only pain intensity significantly higher in the chronic group than acute group, but a significant number of chronic TMD patients reported comorbid musculoskeletal pain in sites 
such as the back, neck and other joints. Thus, the chronic TMD diagnostic protocol must include information about other areas or sites in the body that have been consistently painful.

Psychological factors have been implicated in several aspects of muscular pain and dysfunctional problems as well. Stress-related muscle hyperactivity and parafunctional habits have been suggested as etiologic factors for myogenous pain [18]. Several studies have reported that patients with muscular pain show a higher level of psychological distress accompanied by a higher number of parafunctional habits. Park et al. [18] reported that the myogenous pain of TMD patients showed higher psychological index scores than the arthrogenous pain and mixed pain groups. Lindoth et al. [19] found that masticatory muscle pain subgroup of the chronic TMD patients demonstrated more dysfunctional behavioral profiles and significantly more psychological distress than the intracapsular pain subgroup. The present results showed that the percentage of the TMD_M and TMD_JM were significantly higher in the chronic group than in the acute group. This implies that pain with myogenous components may have a more chronic nature. Although the pathophysiology of headache associated with TMD is not fully understood, it is supposed that chronic TMD symptoms may increase pain sensitivity and may influence the frequency, intensity, and chronicity of headache. Additionally, masticatory muscle pain is potential risk factors of tension-type headache [20]. Our study showed that 63\% of chronic TMD patients complained of headache. We speculated that higher prevalence of myogenous pain in the chronic group than in the acute group might contribute the predominance of those with headaches in the chronic group.

Most people suffering from chronic pain are plagued by sleeping disturbances. Insomnia is often seen as a secondary symptom of chronic pain, because pain and sleep have a bidirectional relationship. A disrupted sleep cycle can exacerbate pain, creating a vicious cycle in which insomnia caused by pain further aggravates pain [5]. Moderateto-severe insomnia (score $\geq 15$ ) according to the ISI is frequently (53\%-65\%) reported among patients with chronic pain [21]. In this study the number of patients who reported sleep disturbances due to pain was higher for chronic TMD patients than for acute TMD patients, although this difference was not significant. We also found that the severity of insomnia was significantly higher in chronic TMD patients than in acute TMD patients. Psychological factors, which have a bi-directional relationship with pain, can independently influence sleep quality. Several studies have shown poor sleep complaints to be robustly associated to mood, inactivity, cognitive rumination, and pain-related disability. Emery et al. [9] reported that chronic pain patients with concurrent major depression and insomnia displayed serious pain-related psychosocial impairments. Kim et al. [5] demonstrated that high pain intensity, comorbid musculoskeletal pain, and a high level of depression were all strongly associated with clinical insomnia in patients with chronic neck pain. Dragioti et al. [22] showed that older adults with chronic pain had a high prevalence of clinical insomnia, and anxiety was strongly associated with insomnia. Song et al. [6] found that pain interference and depression were significant predictors of sleep quality in TMD patients. Thus, chronic TMD patients with psychological problems are highly susceptible to insomnia, and insomnia should be considered as an indispensable part of pain management in chronic TMD patients with these risk factors.

Our study has several limitations. First, our study group was relatively small, which may limit the generalizability of our results. Second, we used the self-reported questionnaires to evaluate patients' sleep quality and psychological profiles. Objective measures such as polysomnography and structured clinical interviews were not used. However, patients suspected to have insomnia and psychological impairments in dental clinical practice do not routinely undergo polysomnography and structured clinical interviews, suggesting that our results may more accurately reflect real-life clinical situations.

In conclusion, this study found that the TMD with chronic pain was associated with myogenous pain, clinical insomnia, high psychological distress, and a high level of pain intensity. Therefore, psychological and sleep quality evaluations are mandatory to provide essential data that will allow for improved management of chronic TMD patients, especially in patients suffering from masticatory muscle pain. The present findings suggest that ISI and SRI are simple, valid, and clinically useful tools that can be utilized as a screening device in research related to psychological 
and sleep quality assessments in TMD patients with chronic pain.

\section{CONFLICT OF INTEREST}

No potential conflict of interest relevant to this article was reported.

\section{ORCID}

\author{
Hye-Mi Jeon \\ https://orcid.org/0000-0003-0007-5662 \\ Kyung-Hun Han \\ https://orcid.org/0000-0002-5830-2189 \\ Hye-Min Ju \\ https://orcid.org/0000-0002-9252-6717 \\ Yong-Woo Ahn \\ https://orcid.org/0000-0002-2197-0394 \\ Soo-Min Ok \\ https://orcid.org/0000-0003-1776-371X \\ Sung-Hee Jeong \\ https://orcid.org/0000-0002-6296-4775
}

\section{REFERENCES}

1. Kim HJ, Jang JH, Chung JW. Comparison of clinical symptoms and psychological profiles of temporomandibular joint osteoarthritis between juveniles and adults. J Oral Med Pain 2016;41:4853.

2. Park JH, Shim YJ, Lim HD, Lee YM, Kang JK. Evaluation of anxiety and depression in patients with disc displacement according to diagnostic criteria for temporomandibular disorders. J Oral Med Pain 2018;43:125-130.

3. Laskin DM, Greene CS, Hylander WL. Temporomandibular disorders: an evidence-based approach to diagnosis and treatment. Chicago: Quintessence; 2006. pp. 203-207.

4. Koh KB, Park JK, Kim CH, Cho S. Development of the stress response inventory and its application in clinical practice. Psychosom Med 2001;63:668-678.

5. Kim SH, Lee DH, Yoon KB, An JR, Yoon DM. Factors associated with increased risk for clinical insomnia in patients with chronic neck pain. Pain Physician 2015;18:593-598.

6. Song KW, Kim ME. Sleep quality of patients with temporomandibular disorders: relationship to clinical and psychological characteristics. J Oral Med Pain 2015;40:155-162.

7. Nicassio PM, Moxham EG, Schuman CE, Gevirtz RN. The contri- bution of pain, reported sleep quality, and depressive symptoms to fatigue in fibromyalgia. Pain 2002;100:271-279.

8. Menefee LA, Cohen MJ, Anderson WR, Doghramji K, Frank ED, Lee H. Sleep disturbance and nonmalignant chronic pain: a comprehensive review of the literature. Pain Med 2000;1:156-172.

9. Emery PC, Wilson KG, Kowal J. Major depressive disorder and sleep disturbance in patients with chronic pain. Pain Res Manag 2014;19:35-41.

10. Bastien $\mathrm{CH}$, Vallières A, Morin CM. Validation of the Insomnia Severity Index as an outcome measure for insomnia research. Sleep Med 2001;2:297-307.

11. Cho YW, Song ML, Morin CM. Validation of a Korean version of the insomnia severity index. J Clin Neurol 2014;10:210-215.

12. Laskin DM, Greene CS, Hylander WL. Temporomandibular disorders: an evidence-based approach to diagnosis and treatment. Chicago: Quintessence; 2006. pp. 255-269.

13. Smith GR. The epidemiology and treatment of depression when it coexists with somatoform disorders, somatization, or pain. Gen Hosp Psychiatry 1992;14:265-272.

14. Fishbain DA, Cutler R, Rosomoff HL, Rosomoff RS. Chronic painassociated depression: antecedent or consequence of chronic pain? A review. Clin J Pain 1997;13:116-137.

15. Kaplan GA, Roberts RE, Camacho TC, Coyne JC. Psychosocial predictors of depression. Prospective evidence from the human population laboratory studies. Am J Epidemiol 1987;125:206220.

16. Slack D, Vaux A. Undesirable life events and depression: the role of event appraisals and social support. J Soc Clin Psychol 1988;7:290-296.

17. Hurtig IM, Raak RI, Kendall SA, Gerdle B, Wahren LK. Quantitative sensory testing in fibromyalgia patients and in healthy subjects: identification of subgroups. Clin J Pain 2001;17:316-322.

18. Park JW, Clark GT, Kim YK, Chung JW. Analysis of thermal pain sensitivity and psychological profiles in different subgroups of TMD patients. Int J Oral Maxillofac Surg 2010;39:968-974.

19. Lindroth JE, Schmidt JE, Carlson CR. A comparison between masticatory muscle pain patients and intracapsular pain patients on behavioral and psychosocial domains. J Orofac Pain 2002;16:277-283.

20. Troeltzsch M, Cronin RJ, Brodine AH, Frankenberger R, Messlinger K. Prevalence and association of headaches, temporomandibular joint disorders, and occlusal interferences. J Prosthet Dent 2011;105:410-417.

21. Wiklund T, Linton SJ, Alföldi P, Gerdle B. Is sleep disturbance in patients with chronic pain affected by physical exercise or ACTbased stress management?: a randomized controlled study. BMC Musculoskelet Disord 2018;19:111.

22. Dragioti E, Levin LÅ, Bernfort L, Larsson B, Gerdle B. Insomnia severity and its relationship with demographics, pain features, anxiety, and depression in older adults with and without pain: cross-sectional population-based results from the PainS65+ cohort. Ann Gen Psychiatry 2017;16:15. 